\title{
System Analysis of Relaying with Modulation Diversity
}

\author{
Amir H. Forghani, Georges Kaddoum \\ Department of Electrical Engineering, LaCIME Laboratory \\ University of Quebec, ETS \\ Montreal, Canada \\ Email: pouyaforghani@yahoo.com, georges.kaddoum@etsmtl.ca
}

\author{
Yogesh Nijsure and Francois Gagnon \\ Department of Electrical Engineering, LaCIME Laboratory \\ University of Quebec, ETS \\ Montreal, Canada \\ Email: y.nijsure.2014@ieee.org, francois.gagnon@etsmtl.ca
}

\begin{abstract}
The performance analysis of a relaying system implementing modulation diversity is investigated in this work. Our relaying system is quite novel, since, we assume that the relay always transmits during the relaying phase, i.e., is never silent. Whereas in the other related works assume that the relay first decodes its received signal and upon a successful decoding, transmits to the destination, i.e., the relay is silent upon an unsuccessful decoding. Modulation diversity creates diversity by transforming the angle of a classical modulation to create signal points with distinct components, followed by subsequent interleaving over the components. Assuming transmission over independent Rayleigh fading channels and maximum likelihood detection on the reordered signals at the receiver, the analysis starts with finding the probability density function of the endto-end signal-to-noise (SNR) ratio. Then, a tight upper-bound expression for the error probability is obtained. Following that, we achieve the exact outage probability of the relaying system under-study. Then, exact and upper-bound expressions for the system capacity are presented. Finally, numerical results and comparisons with Monte Carlo simulations are presented.
\end{abstract}

\section{INTRODUCTION}

Significant coding gains over fading channels can be obtained by using modulation diversity (MD). In fact, by using $\mathrm{MD}$, the undesirable impact of the channel fading can be reduced [1], [2]. Current techniques in MD involve mapping the information bits into multiple symbols of a typical constellation, e.g., quadrature phase-shift keying (QPSK), and grouping them into vectors [1], [2], [3]. Then, to distinguish any two vectors by their distinct components, the vectors are rotated by a square spreading matrix. After the rotation, interleaving is applied for transmission via independent fading channels. Hence, the diversity order is determined by the minimum number of distinct components between any two points in the constellation, i.e., minimum Hamming distance. The diversity is obtained at the price of complexity in maximum likelihood (ML) decoding. To reduce complexity, coordinate interleaved space-time codes which take the advantage of combining MD with spatial diversity have been proposed [4], [5]. To achieve spatial diversity without the need of having physical antennas, cooperative techniques can be applied [6], [7]. Indeed, through cooperative communication, relays participate in the signal transmission.

In this paper, we focus on applying modulation diversity in a novel relaying network wherein the relay is always active, i.e., always transmits during the relaying phase, in other words, the relay in this work is never silent. Whereas in other related papers such as [3], it is assumed that the relay decodes its received signal first, and if it decodes its received signal correctly, transmission occurs from the relay to the destination, in other words, the relay is silent upon an unsuccessful decoding. We analyze the system in terms of important performance criteria, namely, error probability, outage probability and capacity. In this work we assume that the relay must decode the source symbols, with some finite error probability, and then create signal from the detected symbols.

The rest of the paper is organized as follows. The system model is described in Section II. In Section III, first, we obtain a tight upper-bound expression for the error probability, then, we find the outage probability in closed-form. The Ergodic capacity and the upper-bound capacity are obtained in Section IV. To verify the accuracy of the analysis, simulation and analytical results along with complementary discussions are provided in Section V. Finally, the paper is concluded in Section VI.

\section{RELAYING With Modulation DiVERsity}

As illustrated in Fig. 1, the modulation diversity (MD)based relaying system consists of a source node, $S$, which communicates with its destination node, $D$, through an intermediate relay, $R$, that always transmits to the destination within the relaying phase. The $S-D, S-R$ and $R-D$ links are assumed to be mutually independent Rayleigh fading channels with gains $\alpha_{S, D}, \alpha_{S, R}$ and $\alpha_{R, D}$, respectively. The additive white Gaussian noise (AWGN) at a receive node is assumed of zero mean and variance $N_{0}$. The concept of modulation diversity implies that the original constellation at the source, QPSK in the case of this paper, is rotated by a transformation angle, $\theta$ [3]. Given two constellation points in the original constellation, say $x_{m}$ and $x_{n}$, their corresponding points in the rotated constellation denoted $\sigma$, are given by $s_{m}=x_{m} \exp (j \theta)$ and $s_{n}=x_{n} \exp (j \theta)$, respectively. The rotation allows the in-phase and quadrature components of the transmit signal to provide enough information to uniquely represent the original signal [8]. In [9], an exhaustive list of the rotation angles for multi-dimensional signal spaces is presented. To transmit the in-phase and quadrature components of each constellation point through independent links, an interleaver is used at the source. The signals after interleaving, denoted $v_{S}$ (the source signal) and $v_{R}$ (the relay signal which will be generated in a subsequent slot), are represented by: 
$v_{S}=\Re\left(s_{m}\right)+j \times \Im\left(s_{n}\right)$ and $v_{R}=\Re\left(s_{n}\right)+j \times \Im\left(s_{m}\right)$, where $\Re\left(s_{m}\right)$ and $\Re\left(s_{n}\right)$ represent the in-phase, and $\Im\left(s_{m}\right)$ and $\Im\left(s_{n}\right)$ represent the quadrature components of $s_{m}$ and $s_{n}$, respectively. Indeed, by interleaving, the constellation is changed to a new constellation. Hence, the signals $v_{S}$ and $v_{R}$ are located at the new constellation, denoted $V$.

In the first time slot, the signal $v_{S}$ is transmitted from the source node. Correspondingly, the signals received at the relay and at the destination node are given by

$$
\begin{gathered}
y_{S, R}=\alpha_{S, R} \sqrt{E_{S}} v_{S}+n_{S, R} \\
y_{S, D}=\alpha_{S, D} \sqrt{E_{S}} v_{S}+n_{S, D},
\end{gathered}
$$

where $E_{S}$ represents the energy of the transmit signal from the source and $n_{S, R}$ and $n_{R, D}$ are the AWGN terms. In the second time slot, transmission of the signal $v_{R}$ from the relay to the destination is performed. The signal received at the destination from the relay can be expressed as

$$
y_{R, D}=\alpha_{R, D} \sqrt{E_{R}} v_{R}+n_{R, D}
$$

where $E_{R}$ denotes the energy of the transmit signal from the relay and $n_{R, D}$ represents the AWGN term in $R-D$ link.

At the destination, the detection is carried out by combining the signals received from the source and the relay. The destination performs reordering on the received components to detect the source signals. The reordered signals can be written as

$$
\begin{aligned}
& r_{1}=\Re\left(y_{S, D}\right)+j \times \Im\left(y_{R, D}\right) \\
& r_{2}=\Re\left(y_{R, D}\right)+j \times \Im\left(y_{S, D}\right) .
\end{aligned}
$$

Finally, ML detector is applied at the receiver to detect the source signals from the reordered signals. The ML detection rule can be formulated as

$$
\begin{aligned}
\hat{s}_{m}= & \arg \min \left(\left|\Re\left(r_{1}\right)-\sqrt{E_{S}} \alpha_{S, D} \Re(s)\right|^{2}\right. \\
& \left.+\left|\Im\left(r_{1}\right)-\sqrt{E_{R}} \alpha_{R, D} \Im(s)\right|^{2}\right) . \\
\hat{s}_{n}= & \arg \min \left(\left|\Re\left(r_{2}\right)-\sqrt{E_{R}} \alpha_{R, D} \Re(s)\right|^{2}\right. \\
+ & \left.\left|\Im\left(r_{2}\right)-\sqrt{E_{S}} \alpha_{S, D} \Im(s)\right|^{2}\right) .
\end{aligned}
$$

\section{ERror And OUtAge Performance}

In this section, upper-bound error probability and following that, outage probability of the MD-based relaying system are assessed. To find the error probability, first, we need to calculate the minimum distance of the rotated constellation, i.e., $d_{\min }$. For any two arbitrary signal points located at the rotated constellation, e.g., $s$ and $\hat{s}, d_{\text {min }}$ is defined by $d_{\min }=\min _{s, \hat{s} \in \sigma}\left(|s-\hat{s}|^{2}\right)^{1}$. For the constant sourcedestination $(S \rightarrow D)$ and relay-destination $(R \rightarrow D)$ channel gains, the conditional pairwise error probability of choosing

\footnotetext{
${ }^{1}$ Calculation of $d_{\min }$ for the considered system is out of scope of this paper, since the authors in [3] have already demonstrated the steps to obtain that.
}

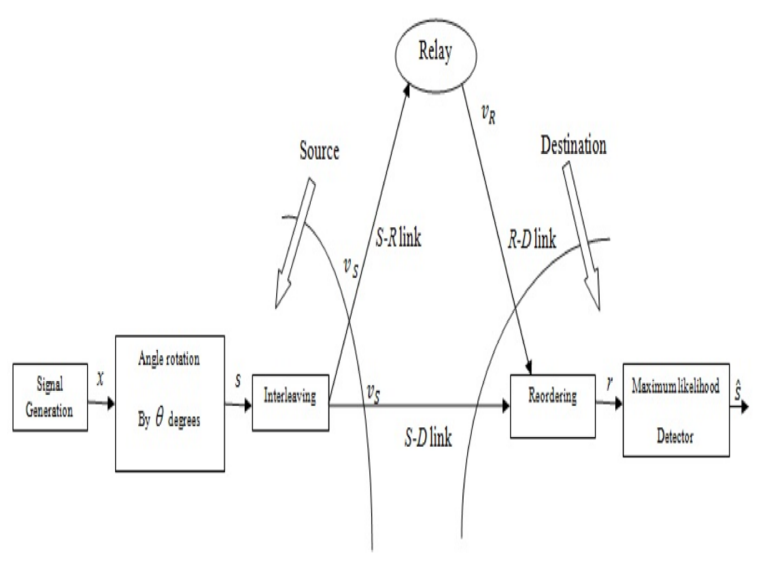

Fig. 1. The MD-based relaying system

$\hat{s}$ at the destination is denoted $\operatorname{CPEP}\left(s, \hat{s} \mid \alpha_{R, D}, \alpha_{S, D}\right)$, considering that $s$ is transmitted. Considering that for any $s$ and $\hat{s}, d_{\min } \leq(\Re(s)-\Re(\hat{s}))^{2}$ and $d_{\min } \leq(\Im(s)-\Im(\hat{s}))^{2}[3$, Eq. $(16,17)]$, then, the conditional pairwise error probability can be tightly upper-bounded as [10, Eq.(13. 12)] as expressed in (5) on the top of next page.

Accordingly, the probability of error under the assumption of constant $S \rightarrow D$ and $R \rightarrow D$ channel gains, defined by $P_{1}(e)=C \times \operatorname{CPEP}\left(s, \hat{s} \mid \alpha_{R, D}, \alpha_{S, D}\right)$, can be tightly upperbounded by using (5) as

$$
P_{1}(e) \leq C \times Q\left(\sqrt{\frac{d_{\min }}{2}\left(\frac{E_{S}}{N_{0}} \alpha_{S, D}^{2}+\frac{E_{R}}{N_{0}} \alpha_{R, D}^{2}\right)}\right),
$$

where the constant $C$ denotes the average of the total number of pairwise error events. For the QPSK modulation with 4 constellation points, the constant $C$ is equal to $C=\frac{2 \times\left(\begin{array}{l}4 \\ 2\end{array}\right)}{4}=3$, where the term $2 \times\left(\begin{array}{l}4 \\ 2\end{array}\right)$ shows the total number of pairwise error events. Remembering that $\alpha_{S, D}$ and $\alpha_{R, D}$ represent the Rayleigh fading coefficients for the $S \rightarrow D$ and $R \rightarrow$ $D$ links. Hence, the probability density function (PDF) of $\frac{E_{S}}{N_{0}} \alpha_{S, D}^{2}+\frac{E_{R}}{N_{0}} \alpha_{R, D}^{2}, f_{\frac{E_{S}}{2 N_{0}} \alpha_{S, D}^{2}}+\frac{E_{R}}{2 N_{0}} \alpha_{R, D}^{2}(\gamma)$, is obtained as (7) as shown below (5) on the top of next page.

with $\mathrm{E}\left(\alpha_{S, D}^{2}\right)$ as the average of the $S \rightarrow D$ channel power and $\mathrm{E}\left(\alpha_{R, D}^{2}\right)$ as the average of the $R \rightarrow D$ channel power. Further, the probability of error, $P(e)$, can be tightly upperbounded by

$$
P(e)=\int_{0}^{\infty} P_{1}(e) \times f_{\frac{E_{S}}{N_{0}} \alpha_{S, D}^{2}}+\frac{E_{R}}{N_{0}} \alpha_{R, D}^{2}(\gamma) d \gamma
$$

Substituting $P_{1}(e)$ and $f_{\frac{E_{S}}{N_{0}}} \alpha_{S, D}^{2}+\frac{E_{R}}{N_{0}} \alpha_{R, D}^{2}(\gamma)$ in (8) by their respective right hand sides obtained in (6) and (7) and taking the integral, a tight upper-bound expression for the error probability is obtained as (9) as indicated below (7) on the top of next page.

Now, we examine the MD-based relaying system in terms of outage probability, $P_{\text {out }}$. Provided that the transmission is carried out in two time slots, the mutual information, $I$, can be obtained by

$$
I=\frac{1}{2} \log _{2}(1+\gamma) \text {. }
$$




$$
\begin{array}{r}
\operatorname{CPEP}\left(s, \hat{s} \mid \alpha_{R, D}, \alpha_{S, D}\right)=Q\left(\sqrt{\frac{E_{S}}{2 N_{0}}\left(\alpha_{S, D}^{2}(\Re(s)-\Re(\hat{s}))^{2}\right)+\frac{E_{R}}{2 N_{0}}\left(\alpha_{R, D}^{2}(\Im(s)-\Im(\hat{s}))^{2}\right)}\right) \\
\leq Q\left(\sqrt{\frac{d_{\min }}{2}\left(\frac{E_{S}}{N_{0}} \alpha_{S, D}^{2}+\frac{E_{R}}{N_{0}} \alpha_{R, D}^{2}\right)}\right)
\end{array}
$$

$$
f_{\frac{E_{S}}{N_{0}} \alpha_{S, D}^{2}+\frac{E_{R}}{N_{0}} \alpha_{R, D}^{2}}(\gamma)=\left\{\begin{array}{l}
\frac{\exp \left(-\frac{\gamma}{\frac{E_{S}}{N_{0}} \mathrm{E}\left(\alpha_{S, D}^{2}\right)}\right)-\exp \left(-\frac{\gamma}{\frac{E_{R}}{N_{0}} \mathrm{E}\left(\alpha_{R, D}^{2}\right)}\right)}{\frac{E_{S}}{N_{0}} \mathrm{E}\left(\alpha_{S, D}^{2}\right)-\frac{E_{R}}{N_{0}} \mathrm{E}\left(\alpha_{R, D}^{2}\right)} \text { if } E_{S} \mathrm{E}\left(\alpha_{S, D}^{2}\right) \neq E_{R} \mathrm{E}\left(\alpha_{R, D}^{2}\right) \\
\frac{\gamma}{\left(\frac{E_{S}}{N_{0}} \mathrm{E}\left(\alpha_{S, D}^{2}\right)\right)^{2}} \exp \left(-\frac{\gamma}{\left(\frac{E_{S}}{N_{0}} \mathrm{E}\left(\alpha_{S, D}^{2}\right)\right)}\right) \text { if } E_{S} \mathrm{E}\left(\alpha_{S, D}^{2}\right)=E_{R} \mathrm{E}\left(\alpha_{R, D}^{2}\right)
\end{array}\right.
$$

$$
P(e) \leq\left\{\begin{array}{l}
\frac{3}{2}+\frac{3}{2\left(\frac{E_{S}}{N_{0}} \mathrm{E}\left(\alpha_{S, D}^{2}\right)-\frac{E_{R}}{N_{0}} \mathrm{E}\left(\alpha_{R, D}^{2}\right)\right)}\left(-\frac{\frac{E_{S}}{N_{0}} \mathrm{E}\left(\alpha_{S, D}^{2}\right)}{\sqrt{1+\frac{4}{d_{\min } \frac{E_{S}}{N_{0}} \mathrm{E}\left(\alpha_{S, D}^{2}\right)}}}+\frac{\frac{E_{R}}{N_{0}} \mathrm{E}\left(\alpha_{R, D}^{2}\right)}{\sqrt{1+\frac{4}{d_{\min } \frac{E_{R}}{N_{0}} \mathrm{E}\left(\alpha_{R, D}^{2}\right)}}}\right) \\
\text { if } E_{S} \mathrm{E}\left(\alpha_{S, D}^{2}\right) \neq E_{R} \mathrm{E}\left(\alpha_{R, D}^{2}\right) \\
\frac{3 E_{S}}{N_{0}} \mathrm{E}\left(\alpha_{S, D}^{2}\right)\left(\frac{1}{2}-\frac{1}{2 \sqrt{1+\frac{4}{d_{\min } \frac{E_{S}}{N_{0}} \mathrm{E}\left(\alpha_{S, D}^{2}\right)}}}\right) \\
\text { if } E_{S} \mathrm{E}\left(\alpha_{S, D}^{2}\right)=E_{R} \mathrm{E}\left(\alpha_{R, D}^{2}\right)
\end{array}\right.
$$

The outage probability is the probability that $I<r$ or $\gamma<\gamma_{\mathrm{th}}$, where $\gamma_{\text {th }}=2^{2 r}-1$ is the threshold signal-to-noise ratio (SNR) and $r$ is the given rate. Hence, the outage probability is given by

$$
P_{\text {out }}=\int_{0}^{\gamma_{\mathrm{th}}} f_{\frac{E_{S}}{N_{0}} \alpha_{S, D}^{2}+\frac{E_{R}}{N_{0}} \alpha_{R, D}^{2}}(\gamma) d \gamma .
$$

Using (7) and taking the above integral, $P_{\text {out }}$ is obtained as (12) as shown below (9) on the top of next page.

\section{ERGODIC AND UPPER-BOUND CAPACITIES}

In this section, we assess the MD-based relaying system with respect to capacity. We provide closed-form expressions for the Ergodic and upper-bound capacities. The Ergodic capacity denoted $C_{\text {Ergodic }}$, is defined by $C_{\text {Ergodic }}=$ $\frac{1}{2} \mathrm{E}\left(\log _{2}(1+\gamma)\right)$. Accordingly, the capacity can be written in integral form as

$$
C_{\text {Ergodic }}=\frac{1}{2} \int_{0}^{\infty} \log _{2}(1+\gamma) f_{\frac{E_{S}}{N_{0}} \alpha_{S, D}^{2}+\frac{E_{R}}{N_{0}} \alpha_{R, D}^{2}}(\gamma) d \gamma .
$$

By replacing $f_{\frac{E_{S}}{N_{0}} \alpha_{S, D}^{2}+\frac{E_{R}}{N_{0}} \alpha_{R, D}^{2}}(\gamma)$ with the value obtained in (7) and taking the integral, the integral is solved. Thus, yielding (14) as written on the top of page 4, where $\mathrm{E}_{\mathrm{i}}(x)=$ $\int_{-\infty}^{x} \frac{\exp (t)}{t} d t$ represents the Exponential integral. To achieve an insightful expression for the capacity, we obtain the upperbound capacity, $C_{\text {up }}$, by resorting to Jensen's inequality. The upper-bound capacity defined by $C_{\mathrm{up}}=\frac{1}{2} \log _{2}(1+\mathrm{E}(\gamma))$, is obtained as

$$
C_{\text {up }}=\frac{1}{2} \log _{2}\left(1+\frac{E_{S}}{N_{0}} \mathrm{E}\left(\alpha_{S, D}^{2}\right)+\frac{E_{R}}{N_{0}} \mathrm{E}\left(\alpha_{R, D}^{2}\right)\right) .
$$

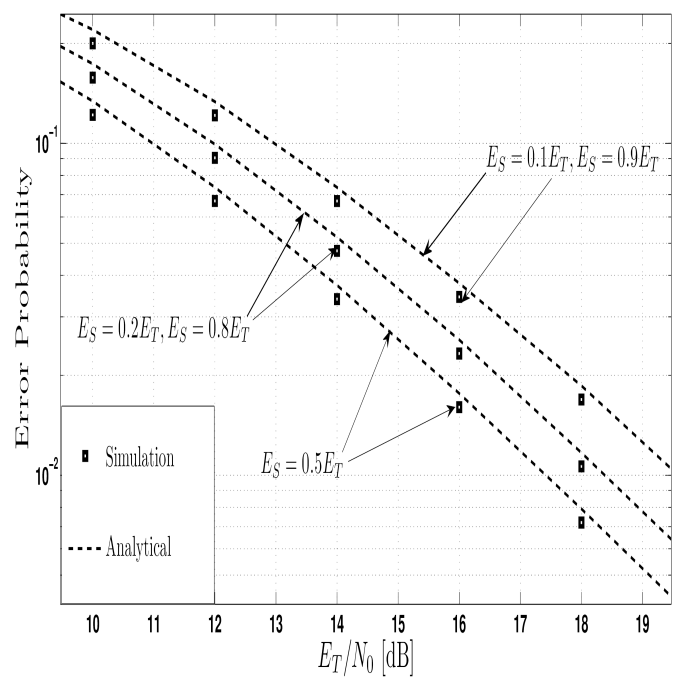

Fig. 2. Error probability curves of the MD-based relaying system for various source energies of $E_{S}=0.1,0.2,0.5,0.8$ and $0.9 E_{T}$.

\section{NumericAl RESUlts}

In this section, figures pertaining to the error probability, outage probability and Ergodic capacity are illustrated. In addition to numerical results, Monte Carlo simulation results are also provided to verify the correctness of the analysis. For the figures, the $X$ axis represents the total available power, $E_{T}$, considering that $E_{T}$ is equal to $E_{S}+E_{R}$. We set the values of $\mathrm{E}\left(\alpha_{S, D}^{2}\right), \mathrm{E}\left(\alpha_{S, R}^{2}\right)$ and $\mathrm{E}\left(\alpha_{R, D}^{2}\right)$ to two, three, and two respectively. For the considered values of $\mathrm{E}\left(\alpha_{S, D}^{2}\right)$ and $\mathrm{E}\left(\alpha_{R, D}^{2}\right)$, the error probability, outage probability and 


$$
\begin{aligned}
& P_{\text {out }}=\left\{\begin{array}{l}
\left.1-\frac{E_{S} \mathrm{E}\left(\alpha_{S, D}^{2}\right) \exp \left(-\frac{\gamma_{\text {th }}}{\frac{E_{S}}{N_{0}} \mathrm{E}\left(\alpha_{S, D}^{2}\right)}\right)-E_{R} \mathrm{E}\left(\alpha_{R, D}^{2}\right) \exp \left(-\frac{\gamma_{\text {th }}}{E_{R}}\right)}{E_{S} \mathrm{E}\left(\alpha_{S, D}^{2}\right)-E_{R} \mathrm{E}\left(\alpha_{R, D}^{2}\right)}\right) \\
1-\exp \left(-\frac{\gamma_{\text {th }}}{\left(\frac{E_{S}}{N_{0}} \mathrm{E}\left(\alpha_{S, D}^{2}\right)\right)}\right) \frac{\left(\frac{E_{S}}{N_{0}} \mathrm{E}\left(\alpha_{S, D}^{2}\right)+\gamma_{\text {th }}\right)}{\frac{E_{S}}{N_{0}} \mathrm{E}\left(\alpha_{S, D}^{2}\right)} \text { if } E_{S} \mathrm{E}\left(\alpha_{S, D}^{2}\right)=E_{R} \mathrm{E}\left(\alpha_{S, D}^{2}\right) \neq E_{R, D}^{2} \mathrm{E}\left(\alpha_{R, D}^{2}\right)
\end{array}\right. \\
& C_{\text {Ergodic }}=\frac{1}{2 \ln (2)} \\
& \times\left\{\begin{array}{c}
\frac{1}{\frac{E_{S}}{N_{0}} \mathrm{E}\left(\alpha_{S, D}^{2}\right)-\frac{E_{R}}{N_{0}} \mathrm{E}\left(\alpha_{R, D}^{2}\right)} \times\left(\begin{array}{l}
-\frac{E_{S}}{N_{0}} \mathrm{E}\left(\alpha_{S, D}^{2}\right) \exp \left(\frac{1}{\frac{E_{S}}{N_{0}} \mathrm{E}\left(\alpha_{S, D}^{2}\right)}\right) \mathrm{E}_{\mathrm{i}}\left(-\frac{1}{\frac{E_{S}}{N_{0}} \mathrm{E}\left(\alpha_{S, D}^{2}\right)}\right)+ \\
\frac{E_{R}}{N_{0}} \mathrm{E}\left(\alpha_{R, D}^{2}\right) \exp \left(\frac{1}{\frac{E_{R}}{N_{0}} \mathrm{E}\left(\alpha_{R, D}^{2}\right)}\right) \mathrm{E}_{\mathrm{i}}\left(-\frac{1}{\frac{E_{R}}{N_{0}} \mathrm{E}\left(\alpha_{R, D}^{2}\right)}\right)
\end{array}\right) \mathrm{E}^{\operatorname{if} E_{S} \mathrm{E}\left(\alpha_{S, D}^{2}\right) \neq E_{R} \mathrm{E}\left(\alpha_{R, D}^{2}\right)} \\
\left(\frac{1}{\frac{E_{S}}{\frac{E}{N}_{0}} \mathrm{E}\left(\alpha_{S, D}^{2}\right)} \exp \left(\frac{1}{\frac{E_{S}}{E_{0}} \mathrm{E}\left(\alpha_{S, D}^{2}\right)}\right) \mathrm{E}_{\mathrm{i}}\left(-\frac{1}{\frac{E_{S}}{N_{0}} \mathrm{E}\left(\alpha_{S, D}^{2}\right)}\right)-\exp \left(\frac{1}{\frac{E_{S}}{N_{0}} \mathrm{E}\left(\alpha_{S, D}^{2}\right)}\right) \mathrm{E}_{\mathrm{i}}\left(-\frac{1}{\frac{E_{S}}{N_{0}} \mathrm{E}\left(\alpha_{S, D}^{2}\right)}\right)+1\right) \\
\text { if } E_{S} \mathrm{E}\left(\alpha_{S, D}^{2}\right)=E_{R} \mathrm{E}\left(\alpha_{R, D}^{2}\right)
\end{array}\right.
\end{aligned}
$$

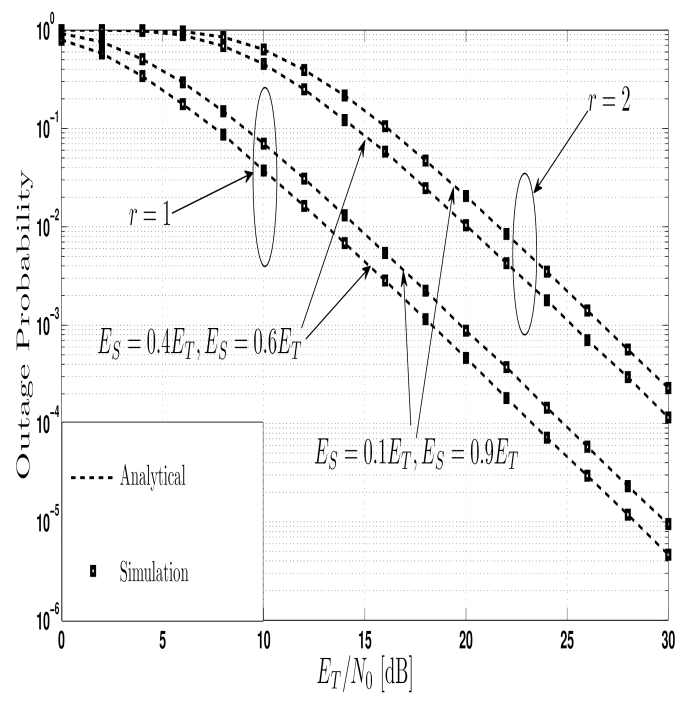

Fig. 3. Outage probability of the MD-based relaying system, considering various source energies of $E_{S}=0.1,0.4,0.6$ and $0.9 E_{T}$ in different rates, $r=1$ and 2 .

Ergodic capacity values are the same for $E_{S}=a E_{T}$ and $E_{S}=(1-a) E_{T}$, where $a$ is a constant between zero and 1. This is due to the fact that the obtained expressions for the error probability, outage probability and Ergodic capacity are symmetric at around $E_{S}=0.5 E_{T}$. Fig. 2 shows the error probability of the MD-based relaying system according to (9) in the range of 9 to $19 \mathrm{~dB}$. The curves are pertinent to the source energies of $E_{S}=0.1,0.2,0.5,0.8$ and $0.9 E_{T}$, respectively. As observed, when $E_{S}=0.2$ and $0.8 E_{T}$, the error performances are better than the cases with $E_{S}=0.1$ and $0.9 E_{T}$, however, the curve pertaining to the $E_{S}=0.5 E_{T}$ outperforms all the other curves. Fig. 3 depicts the outage probability based on (12) assuming different source energies, $E_{S}=0.1,0.4,0.6$ and $0.9 E_{T}$, in the rates $r=1$ and 2 . As seen, when $E_{S}=0.4$ and $0.6 E_{T}$, the outage performances are better than the cases with $E_{S}=0.1$ and $0.9 E_{T}$. As expected,

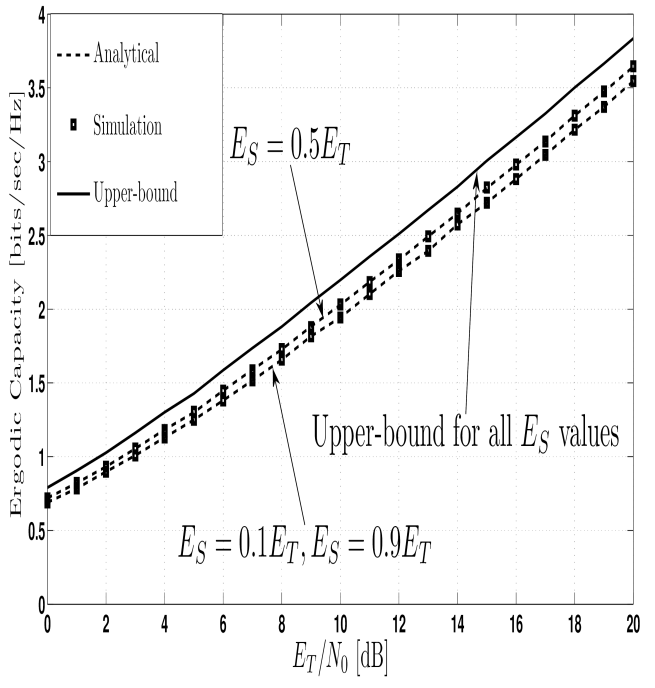

Fig. 4. Ergodic capacity in the source energies of $E_{S}=0.1,0.5$ and $0.9 E_{T}$ along with upper-bound capacity.

there is also a complete agreement between simulation and analytical results as the analytical outage probability is exact. It is verified by the figure that by increasing the rate from $r=1$ to $r=2$, the outage probability increases significantly. In Fig. 4, the curves pertaining to the Ergodic and upperbound capacities according to (14) and (15) are illustrated in bits $/ \mathrm{sec} / \mathrm{Hz}$. The energies of the source are considered $E_{S}=0.1,0.5$ and $0.9 E_{T}$, and as observed the highest capacity is obtained for $E_{S}=0.5 E_{T}$. Also, as expected, the capacity for $E_{S}=0.1 E_{T}$ is equal to the one for $E_{S}=0.9 E_{T}$. This is because of the fact that for the given average $S \rightarrow R, R \rightarrow D$ and $S \rightarrow D$ channel powers, the capacity function has its maximum in $E_{S}=0.5 E_{T}$ and also is symmetric at around $E_{S}=0.5 E_{T}$. Besides, the coincidence between the numerical and simulation results is due to the fact that the analytical capacity is exact. 


\section{CONCluding Remarks}

This paper investigated a system utilizing the combination of modulation diversity and relaying, namely the MD-based relaying system while all the channels are subject to the Rayleigh fading. Such system is quite novel, since, the relay always transmits during the relaying phase, i.e., the relay is never silent. Whereas in the other corresponding papers, it is always assumed that the relay first decodes its received signal and upon a successful decoding, transmits to the destination, i.e., the relay is silent upon an unsuccessful decoding. A tight upper-bound expression for the error probability was obtained. Also, we achieved an exact closed-form expression for the outage probability. Following that, exact Ergodic capacity and upper-bound capacity were found. Finally, illustrative results were depicted to validate the authenticity of the analysis. The system is potentially capable of being developed to a generalized system with multiple relays.

\section{REFERENCES}

[1] E. B. X. Giraud and J. C. Belfiore, "Algebraic tools to build modulation schemes for fading channels," IEEE Transactions on Inform. Theory, vol. 43, pp. 938-952, 1997.

[2] K. Boulle and J. C. Belfiore, "Modulation schemes designed for the Rayleigh fading channel," in Conf. Inform. Sci. and Sys. (CISS?92), Princeton, USA, 1992.

[3] S. A. M. S. A. Ahmadzadeh and A. K. Khandani, "Signal space cooperative communication for single relay model," Ph.D. dissertation, Department of ECE, University of Waterloo, Waterloo, Canada, 2009.

[4] T. E. H. M. Janani, A. Hedayat and A. Nosratinia, "Coded cooperation in wireless communications: space-time transmission and iterative decoding," IEEE Transactions on Signal Processing, vol. 52, pp. 362-371, 2004.

[5] J. Hagenauer, "Rate-compatible punctured convolutional codes (RCPC codes) and their applications," IEEE Transactions on Communications, vol. 36, pp. 389-400, 1988.

[6] S. S. Ikki and M. H. Ahmed, "Performance analysis of cooperative diversity wireless networks over Nakagami-m fading channel," IEEE Commun. Letters, vol. 11, pp. 334-336, 2007.

[7] L. L. Yang and H. H. Chen, "Error probability of digital communications using relay diversity over Nakagami-m fading channels," IEEE Transactions on Wireless Communications, vol. 7, pp. 1806-1811, 2008.

[8] J. Boutros and E. Viterbo, "Signal space diversity: a power- and bandwidth-efficient diversity technique for the Rayleigh fading channel," IEEE Transactions on Inform. Theory, vol. 44, pp. 1453-1467, 1998.

[9] E. Viterbo and F. Oggier. Tables of algebraic rotations. [Online] Available: http://www.tlc.polito.it / viterbo/rotations/rotations.html

[10] M. K. Simon and M.-S. Alouini, Digital Communication over Fading Channels, 2nd edition. John Wiley and Sons Inc., 2005. 